\title{
CLASSICAL OPERATORS ON WEIGHTED BANACH SPACES OF ENTIRE FUNCTIONS
}

\author{
MARÍA J. BELTRÁN, JOSÉ BONET, AND CARMEN FERNÁNDEZ
}

(Communicated by Thomas Schlumprecht)

\begin{abstract}
We study the operators of differentiation and of integration and the Hardy operator on weighted Banach spaces of entire functions. We estimate the norm of the operators, study the spectrum, and analyze when they are surjective, power bounded, hypercyclic, and (uniformly) mean ergodic.
\end{abstract}

\section{INTRODUCTION AND NOTATION}

The aim of this paper is to study the following three operators on weighted spaces of entire functions defined by means of sup norms: the differentiation operator $\operatorname{Df}(z)=f^{\prime}(z)$, the integration operator $J f(z)=\int_{0}^{z} f(\zeta) d \zeta$, and the Hardy operator $\operatorname{Hf}(z)=\frac{1}{z} \int_{0}^{z} f(\zeta) d \zeta, z \in \mathbb{C}$. The continuity of the differentiation and the integration operators between weighted Banach spaces of holomorphic functions has been studied by Harutyunyan and Lusky [13. The continuity of these two operators on weighted Banach spaces of entire functions associated to a weight $v$ is determined by the growth or decline of $v(r) e^{\alpha r}$ for some $\alpha>0$ in an interval $\left[r_{0}, \infty[\right.$. The surjectivity and the spectrum of the differentiation operator on weighted Banach spaces of entire functions were studied by Atzmon and Brive [2]. Although there is a huge literature on the Hardy operator on different function spaces (see e.g. 1]), it seems that it has not yet been studied in the context considered in our paper. Bonet [8] (see also [9]) investigated when the operator of differentiation is hypercyclic or chaotic on weighted Banach spaces of entire functions. It is our purpose to continue this work by analyzing other operators as well as other properties related to the dynamical behaviour of the operator, such as being power bounded or mean ergodic, thus also complementing work by Bonet and Ricker [10] about mean ergodic multiplication operators.

A continuous and linear operator $T$ from a Banach space $E$ into itself is called power bounded if the sequence of its iterates $\left(T^{n}\right)_{n \in \mathbb{N}}$ is equicontinuous. By the uniform boundedness principle this happens if and only if the orbit $\left(x, T x, T^{2} x, \ldots\right)$ is bounded for every $x \in E$. The operator $T \in L(E)$ is called hypercyclic if there is $x \in E$ with a dense orbit. We refer the reader to the recent books by Bayart and Matheron [3] and by Grosse-Erdmann and Peris [12 for linear dynamics. The

Received by the editors December 5, 2011 and, in revised form, February 3, 2012.

2010 Mathematics Subject Classification. Primary 47B38; Secondary 47A16, 46E15.

The authors were partially supported by MEC and FEDER Project MTM2010-15200, by GV Project Prometeo/2008/101, by grant F.P.U. AP2008-00604, and by Conselleria d'Educació de la GVA, Project GV/2010/040. 
operator $T$ is said to be mean ergodic if the limits

$$
P x:=\lim _{N \rightarrow \infty} \frac{1}{N} \sum_{n=1}^{N} T^{n} x, x \in E,
$$

exist in $E$. A power bounded operator $T$ is mean ergodic precisely when $E=$ $\operatorname{Ker}(I-T) \oplus \overline{\operatorname{Im}(I-T)}$. Here $I$ stands for the identity on $E$. If the convergence is in the operator norm, the operator is called uniformly mean ergodic. Clearly, if $T$ is mean ergodic, then $\lim _{n \rightarrow \infty}\left\|T^{n} x\right\| / n=0$ for each $x \in E$, and if it is uniformly mean ergodic, $\lim _{n \rightarrow \infty}\left\|T^{n}\right\| / n=0$. If this condition is satisfied, the operator $T$ is uniformly mean ergodic if and only if $\operatorname{Im}(I-T)$ is closed [16]. An operator $T$ is said to be quasi-compact if $T^{n}$ is compact for some $n \in N$. Quasi-compact operators share some properties of compact operators, in particular the spectrum $\sigma(T)$ of a quasi-compact operator $T$ reduces to its eigenvalues and $\{0\}$. Our notation for functional analysis and operator theory is standard. We refer the reader e.g. to [21] and 23. For ergodic theory of operators on Banach spaces, see 15 .

In what follows $\mathcal{H}(\mathbb{C})$ and $\mathcal{P}$ will denote the spaces of entire functions and of polynomials, respectively. The space $\mathcal{H}(\mathbb{C})$ will be endowed with the compact open topology $\tau_{c o}$. It is easy to see that the three operators $D, J$ and $H$ are continuous on $\mathcal{H}(\mathbb{C})$.

Throughout the paper, a weight $v$ is a continuous function $v:[0,+\infty[\rightarrow] 0,+\infty[$ which is non-increasing on $\left[0, \infty\right.$ [ and satisfies $\lim _{r \rightarrow \infty} r^{m} v(r)=0$ for each $m \in \mathbb{N}$. For such a weight, the weighted Banach spaces of entire functions are defined by

$$
\begin{gathered}
H_{v}^{\infty}(\mathbb{C}):=\left\{f \in \mathcal{H}(\mathbb{C})\left|\|f\|_{v}:=\sup _{z \in \mathbb{C}} v(|z|)\right| f(z) \mid<+\infty\right\}, \\
H_{v}^{0}(\mathbb{C}):=\left\{f \in \mathcal{H}(\mathbb{C})\left|\lim _{|z| \rightarrow \infty} v(|z|)\right| f(z) \mid=0\right\},
\end{gathered}
$$

endowed with the sup norm $\|\cdot\|_{v}$. Clearly $H_{v}^{0}(\mathbb{C})$ is a closed subspace of $H_{v}^{\infty}(\mathbb{C})$ which contains the polynomials. Both are Banach spaces and the closed unit ball of $H_{v}^{\infty}(\mathbb{C})$ is $\tau_{c o}$-compact. The polynomials are contained and dense in $H_{v}^{0}(\mathbb{C})$, but the monomials are not in general a Schauder basis [19]. The Cesàro means of the Taylor polynomials satisfy $\left\|C_{n} f\right\|_{v} \leq\|f\|_{v}$ for each $f \in H_{v}^{\infty}(\mathbb{C})$, and the sequence $\left(C_{n} f\right)_{n}$ is \|\|$_{v}$-convergent to $f$ when $f \in H_{v}^{0}(\mathbb{C})$ [4. Clearly, changing the value of $v$ on a compact interval does not change the spaces and gives an equivalent norm. By [6, Ex 2.2] the bidual of $H_{v}^{0}(\mathbb{C})$ is isometrically isomorphic to $H_{v}^{\infty}(\mathbb{C})$. When $v(r)=e^{-\alpha r}(\alpha>0)$, we write $H_{\alpha}^{\infty}(\mathbb{C})$ and $H_{\alpha}^{0}(\mathbb{C})$ for the weighted Banach spaces and denote their norm by \|\|$_{\alpha}$. The spaces $H_{a, \alpha}^{\infty}(\mathbb{C})$ and $H_{a, \alpha}^{0}(\mathbb{C})(\alpha>0, a \in \mathbb{R})$ correspond to the following weights: $v(r)=e^{-\alpha}, r \in\left[0,1\left[, v(r)=r^{a} e^{-\alpha r}, r \geq 1\right.\right.$, if $a<0$ and $v(r)=(a / \alpha)^{a} e^{-a}, r \in\left[0, a / \alpha\left[, v(r)=r^{a} e^{-\alpha r}, r \geq a / \alpha\right.\right.$, if $a>0$. In this case the norm will be denoted by \|\|$_{a, \alpha}$. Spaces of this type appear in the study of growth conditions of analytic functions and have been investigated in various articles; see e.g. [4, 5, 7, 11, 19, 20, and the references therein.

Our main theorem summarizes our results for the spaces $H_{\alpha}^{\infty}(\mathbb{C})$ and $H_{\alpha}^{0}(\mathbb{C})$, where $v(r)=e^{-\alpha r}, \alpha>0$.

Theorem 1.1. (1) The differentiation operator $D$ satisfies $\left\|D^{n}\right\|_{\alpha}=n !\left(\frac{e \alpha}{n}\right)^{n}$ for each $n \in \mathbb{N}$; hence it is power bounded if and only if $\alpha<1$. The spectrum of $D$ is the closed disc of radius $\alpha$. It is uniformly mean ergodic on $H_{\alpha}^{\infty}(\mathbb{C})$ and $H_{\alpha}^{0}(\mathbb{C})$ if $\alpha<1$ and not mean ergodic if $\alpha>1$, and it is not mean ergodic on $H_{1}^{\infty}(\mathbb{C})$ and not uniformly mean ergodic on $H_{1}^{0}(\mathbb{C})$. 
(2) The integration operator $J$ is never hypercyclic on $H_{\alpha}^{0}(\mathbb{C})$, and it satisfies $\left\|J^{n}\right\|_{\alpha}=1 / \alpha^{n}$ for each $n \in \mathbb{N}$. Hence, it is power bounded if and only if $\alpha \geq 1$. The spectrum of $J$ is the closed disc of radius $1 / \alpha$. If $\alpha>1$, then $J$ is uniformly mean ergodic on $H_{\alpha}^{\infty}(\mathbb{C})$ and $H_{\alpha}^{0}(\mathbb{C})$, and it is not mean ergodic on these spaces if $\alpha<1$. If $\alpha=1$, then $J$ is not mean ergodic on $H_{1}^{\infty}(\mathbb{C})$, and mean ergodic but not uniformly mean ergodic on $H_{1}^{0}(\mathbb{C})$.

(3) The Hardy operator $H$ is compact and has norm 1, its spectrum coincides with the set $\{1 / n\}_{n \in \mathbb{N}} \cup\{0\}$, and it is power bounded and uniformly mean ergodic on $H_{\alpha}^{\infty}(\mathbb{C})$ and $H_{\alpha}^{0}(\mathbb{C})$ for all $\alpha>0$. Therefore, it is not hypercyclic on $H_{\alpha}^{0}(\mathbb{C})$.

It follows from [8, Corollary 2.6] that the differentiation operator on $H_{\alpha}^{0}(\mathbb{C})$ is not hypercyclic and has no periodic point different from 0 if $\alpha<1$, it is hypercyclic and has a dense set of periodic points if $\alpha>1$, and it is hypercyclic but has no periodic point different from 0 if $\alpha=1$.

\section{Preliminaries}

Our first result is inspired by [8, Proposition 1.1].

Lemma 2.1. Let $T:\left(\mathcal{H}(\mathbb{C}), \tau_{c o}\right) \rightarrow\left(\mathcal{H}(\mathbb{C}), \tau_{c o}\right)$ be a continuous linear operator such that $T(\mathcal{P}) \subset \mathcal{P}$. The following conditions are equivalent:

(i) $T\left(H_{v}^{\infty}(\mathbb{C})\right) \subset H_{v}^{\infty}(\mathbb{C})$,

(ii) $T: H_{v}^{\infty}(\mathbb{C}) \rightarrow H_{v}^{\infty}(\mathbb{C})$ is continuous,

(iii) $T\left(H_{v}^{0}(\mathbb{C})\right) \subset H_{v}^{0}(\mathbb{C})$,

(iv) $T: H_{v}^{0}(\mathbb{C}) \rightarrow H_{v}^{0}(\mathbb{C})$ is continuous.

Moreover, if (i)-(iv) hold, then $\|T\|_{L\left(H_{v}^{\infty}(\mathbb{C})\right)}=\|T\|_{L\left(H_{v}^{0}(\mathbb{C})\right)}$ and $\sigma_{H_{v}^{\infty}(\mathbb{C})}(T)=$ $\sigma_{H_{v}^{0}(\mathbb{C})}(T)$.

Proof. The equivalences (i)-(ii) and (iii)-(iv) follow from the closed graph theorem. (ii) $\Rightarrow$ (iii) comes easily from the fact that the polynomials are dense in $H_{v}^{0}(\mathbb{C})$, $T(\mathcal{P}) \subset \mathcal{P}$ and $H_{v}^{0}(\mathbb{C})$ is closed in $H_{v}^{\infty}(\mathbb{C})$. Clearly, $\|T\|_{L\left(H_{v}^{0}(\mathbb{C})\right)} \leq\|T\|_{L\left(H_{v}^{\infty}(\mathbb{C})\right)}$. To show (iv) $\Rightarrow$ (ii), observe that given $f \in H_{v}^{\infty}(\mathbb{C})$, the sequence of the Cesàro means of its Taylor polynomials $\left(C_{n} f\right)_{n}$ belongs to $H_{v}^{0}(\mathbb{C})$; therefore $\left\|T\left(C_{n} f\right)\right\|_{v} \leq$ $\|T\|_{L\left(H_{v}^{0}(\mathbb{C})\right)}|| C_{n} f\left\|_{v} \leq\right\| T\left\|_{L\left(H_{v}^{\mathrm{o}}(\mathbb{C})\right)}|| f\right\|_{v}$. Hence, from the $\tau_{c o}$-compactness of the closed unit ball and the $\tau_{c o}$-continuity of $T$, we conclude $\|T f\|_{v} \leq\|T\|_{L\left(H_{v}^{0}(\mathbb{C})\right)}\|f\|_{v}$. The assertion about the spectra is clear as the bi-transpose $T^{\prime \prime}$ of $T: H_{v}^{0}(\mathbb{C}) \rightarrow$ $H_{v}^{0}(\mathbb{C})$ is $T: H_{v}^{\infty}(\mathbb{C}) \rightarrow H_{v}^{\infty}(\mathbb{C})$ by $[4$ and $[6]$. It is a well-known fact that $\sigma(T)=\sigma\left(T^{\prime}\right)=\sigma\left(T^{\prime \prime}\right)$.

As the three operators under consideration satisfy the hypothesis of the previous result, in what follows we will write $\|T\|_{v}$ instead of $\|T\|_{L\left(H_{v}^{\infty}(\mathbb{C})\right)}=\|T\|_{L\left(H_{v}^{0}(\mathbb{C})\right)}$ and $\sigma_{v}(T)$ for the spectrum. The notation $\|T\|_{a, \alpha},\|T\|_{\alpha}, \sigma_{a, \alpha}(T)$, and $\sigma_{\alpha}(T)$ will refer to the cases $v(r)=r^{a} e^{-\alpha r}$ and $v(r)=e^{-\alpha r}$, respectively.

Lemma 2.2. $J-\lambda I$ is injective on $\mathcal{H}(\mathbb{C})$ for each $\lambda \in \mathbb{C}$.

Proof. For $\lambda=0$ this is trivial, since $D J=I$. For $\lambda \neq 0$, the equation $J f-\lambda f=0$ implies $f-\lambda f^{\prime}=0$, hence $f(z)=C e^{z / \lambda}$, and, as $f(0)=\frac{1}{\lambda} J f(0)=0$, we conclude $f=0$.

Proposition 2.3. Let $T=D$ or $T=J$ and assume that $T: H_{v}^{\infty}(\mathbb{C}) \rightarrow H_{v}^{\infty}(\mathbb{C})$ is continuous. The following conditions are equivalent: 
(i) $T: H_{v}^{\infty}(\mathbb{C}) \rightarrow H_{v}^{\infty}(\mathbb{C})$ is uniformly mean ergodic,

(ii) $T: H_{v}^{0}(\mathbb{C}) \rightarrow H_{v}^{0}(\mathbb{C})$ is uniformly mean ergodic,

(iii) $\lim _{N \rightarrow \infty} \frac{\left\|T+\cdots+T^{N}\right\|_{v}}{N}=0$.

Moreover, if $1 \in \sigma_{v}(T)$, then $T$ is not uniformly mean ergodic.

Proof. The implications (i) $\Leftrightarrow$ (ii) and (iii) $\Rightarrow$ (i) are clear from Lemma 2.1. We show (ii) $\Rightarrow$ (iii).

Suppose first that $T=D$ is uniformly mean ergodic on $H_{v}^{0}(\mathbb{C})$. Since the polynomials are dense and the sequence $\left(\frac{1}{N} \sum_{n=1}^{N} D^{n}\right)_{N}$ converges pointwise to zero on $\mathcal{P}$, we have that

$$
\lim _{N \rightarrow \infty} \frac{1}{N}\left\|\sum_{n=1}^{N} D^{n}\right\|_{v}=0 .
$$

For $T=J$, we only have to prove that $\lim _{N} \frac{\left(J+\cdots+J^{N}\right)(f)}{N}=0$ for each $f \in H_{v}^{0}(\mathbb{C})$. By assumption, the $\operatorname{limit}_{N} \frac{\left(J+\cdots+J^{N}\right)(f)}{N}$ exists. Moreover,

$$
\begin{aligned}
J\left(\lim _{N} \frac{\left(J+\cdots+J^{N}\right)(f)}{N}\right) & =\lim _{N}\left(\frac{\left(J+\cdots+J^{N+1}\right)(f)}{N+1} \frac{N+1}{N}-\frac{J f}{N}\right) \\
& =\lim _{N} \frac{\left(J+\cdots+J^{N}\right)(f)}{N} .
\end{aligned}
$$

By Lemma 2.2, the conclusion follows.

If $T$ is uniformly mean ergodic, since $\lim _{N} \frac{\left\|T+\cdots+T^{N}\right\|}{N}=0$, for $N$ big enough the operator $I-\frac{1}{N} \sum_{n=1}^{N} T^{n}$ is invertible, i.e., $N \notin \sigma_{v}(p(T))$ for $p(z)=\sum_{n=1}^{N} z^{n}$, which, by the spectral mapping theorem, coincides with $p\left(\sigma_{v}(T)\right)$. Thus, $1 \notin \sigma_{v}(T)$.

The proof of Proposition 2.3 in fact shows that if $J$ is mean ergodic on $H_{v}^{\infty}(\mathbb{C})$ or on $H_{v}^{0}(\mathbb{C})$, then $\lim _{N} \frac{\left(J+\cdots+J^{N}\right)(f)}{N}=0$ for every $f$ in the corresponding space.

An operator $T$ on a Banach space $X$ is said to be Cesàro power bounded if the sequence of the Cesàro means of its iterates is equicontinuous. By Lemma 2.1 and the Banach-Steinhaus theorem, $D$ is Cesàro power bounded on $H_{v}^{\infty}(\mathbb{C})$ if and only if it is Cesàro power bounded on $H_{v}^{0}(\mathbb{C})$. Since the polynomials are dense in $H_{v}^{0}(\mathbb{C})$, the operator $D$ is mean ergodic on $H_{v}^{0}(\mathbb{C})$ if and only if it is Cesàro power bounded. In this case, $P(f)=0$ for every $f \in H_{v}^{0}(\mathbb{C})$.

The norms of the monomials played an important role in the characterization of hypercyclic differentiation operators in [8]. They are also important now.

Lemma 2.4. Let $v$ be a weight such that the differentiation operator $D$ and the integration operator $J$ are continuous on $H_{v}^{0}(\mathbb{C})$ and on $H_{v}^{\infty}(\mathbb{C})$.

(i) If $D$ is power bounded (resp. uniformly mean ergodic) on $H_{v}^{\infty}(\mathbb{C})$, then $\inf \left(\frac{\left\|z^{n}\right\|_{v}}{n !}\right)_{n}>0$ (resp. $\left(\frac{\left\|z^{n}\right\|_{v}}{(n-1) !}\right)_{n}$ tends to infinity).

(ii) If $J$ is power bounded (resp. mean ergodic) on $H_{v}^{\infty}(\mathbb{C})$, then $\left(\frac{\left\|z^{n}\right\|_{v}}{n !}\right)_{n}$ is bounded (resp. $\left(\frac{\left\|z^{n}\right\|_{v}}{n ! n}\right)_{n}$ tends to zero).

Proof. This follows easily from the inequalities

$$
v(0) n ! \leq\left\|D^{n}\right\|_{v}\left\|z^{n}\right\|_{v}
$$

and

$$
v(0)\left\|J^{n}\right\|_{v} \geq\left\|J^{n}(1)\right\|_{v}=\frac{\left\|z^{n}\right\|_{v}}{n !} .
$$


For weights $v(r)=r^{a} e^{-\alpha r}(\alpha>0, a \in \mathbb{R})$ for $r \geq r_{0}$ for some $r_{0} \geq 0$, we have

$$
\left\|z^{n}\right\|_{v} \approx\left(\frac{n+a}{e \alpha}\right)^{n+a}
$$

with equality for $v(r)=e^{-\alpha r}, r \geq 0$. It is enough to estimate the maximum of the function $g(r)=r^{n+a} e^{-\alpha r}$ and to have in mind that the weight $v$ may change in a compact interval.

\section{MAin RESUlts}

\subsection{The integration operator.}

Proposition 3.1. The operator $J$ is never hypercyclic on $H_{v}^{0}(\mathbb{C})$, and it has no periodic points different from 0 in $H_{v}^{\infty}(\mathbb{C})$.

Proof. By the very definition, $J f(0)=0$ for every $f \in H_{v}^{0}(\mathbb{C})$. Therefore, $\operatorname{Im}(J)$, and a fortiori the orbit of an element, cannot be dense. Now suppose that $J^{n} f=f$ for some $f \neq 0$ and some $n \in \mathbb{N}$. We have $f-D^{n} f=0$; therefore $f$ consists of a linear combination of $e^{\lambda_{k} z}$ where $\lambda_{k}$ are the roots of $\lambda^{n}-1=0$. Then it is easy to see that $J^{n} f=f$ implies $f=0$.

Proposition 3.2. Let $v$ be a weight such that $v(r) e^{\alpha r}$ is non-increasing for some $\alpha>0$. Then, $J$ is continuous with $\|J\|_{v} \leq 1 / \alpha$. In particular, $\sigma_{v}(J) \subset(1 / \alpha) \overline{\mathbb{D}}$. Moreover, $\left\|J^{n}\right\|_{\alpha}=1 / \alpha^{n}$ for all $n \in \mathbb{N}_{0}$.

Proof. Fix $f \in H_{v}^{\infty}(\mathbb{C})$. We have

$$
v(|z|)|J f(z)| \leq|z| \int_{0}^{1} v(t|z|)|f(t z)| \exp (\alpha(t-1)|z|) d t \leq\|f\|_{v} \frac{1}{\alpha} .
$$

Therefore $\left\|J^{n}\right\|_{v} \leq 1 / \alpha^{n}$. The statement about $\sigma_{v}(J)$ follows immediately.

On the other hand, for $v(r)=e^{-\alpha r}$, we have

$$
\left\|J^{n}\right\|_{\alpha} \geq \sup _{k \in \mathbb{N}} \frac{\left\|J^{n}\left(z^{k}\right)\right\|_{\alpha}}{\left\|z^{k}\right\|_{\alpha}} \geq \sup _{k \in \mathbb{N}} \frac{k !}{(k+n) !} \frac{\left\|z^{k+n}\right\|_{\alpha}}{\left\|z^{k}\right\|_{\alpha}} .
$$

We can apply the Stirling formula to conclude

$$
\left\|J^{n}\right\|_{\alpha} \geq \sup _{k \in \mathbb{N}} \frac{k !}{(k+n) !} \frac{(e \alpha / k)^{k}}{(e \alpha /(k+n))^{k+n}} \geq \sup _{k \in \mathbb{N}} \frac{(1+n / k)^{k}}{\alpha^{n} e^{n}} \geq \frac{1}{\alpha^{n}} .
$$

Proposition 3.3. Let $v$ be a weight such that $J$ is continuous on $H_{v}^{\infty}(\mathbb{C})$, and assume that $v(r) e^{\alpha r}$ is increasing. Then, $\sigma_{v}(J) \supset(1 / \alpha) \mathbb{D}$.

Proof. To see that $(1 / \alpha) \mathbb{D} \subset \sigma_{v}(J)$, we show that $J-\lambda I$ is not surjective on $H_{v}^{\infty}(\mathbb{C})$ for $|\lambda|<\frac{1}{\alpha}$. For $\lambda=0, J$ is not surjective on any $H_{v}^{\infty}(\mathbb{C})$ (without any additional assumption) since $J f(0)=0$ for each $f$; hence the equation $J(f)=1$ has no solution. Now assume that $\lambda \neq 0$ and that there is $f \in H_{v}^{\infty}(\mathbb{C})$ such that $J f-\lambda f=1$. Then, $f-\lambda f^{\prime}=0$ and, as $e^{z / \lambda} \notin H_{v}^{\infty}(\mathbb{C})$, we have $f \equiv 0$; therefore $J f-\lambda f \neq 1$.

Corollary 3.4. The spectrum of J satisfies

$$
\sigma_{a, \alpha}(J)=(1 / \alpha) \overline{\mathbb{D}} .
$$


Proof. We can apply Proposition 3.2 to conclude that $J$ is continuous on $H_{a, \alpha}^{\infty}(\mathbb{C})$ and $H_{a, \alpha}^{0}(\mathbb{C})$. By Proposition $3.3, \sigma_{a, \alpha}(J) \supset(1 / \alpha) \mathbb{D}$. On the other hand, for each $\beta<\alpha$, the function $v(r) e^{\beta r}$ is decreasing in some interval $\left[r_{0}, \infty[\right.$. Therefore, for an equivalent norm, $\left\|J^{n}\right\| \leq \frac{1}{\beta^{n}}$, and thus the spectral radius $r(J)$ of $J$, satisfies $r(J) \leq \frac{1}{\beta}$. Since $\beta<\alpha$ is arbitrary, the reverse inclusion holds.

Theorem 3.5. Assume that $J$ is continuous.

(i) $J$ is power bounded on $H_{v}^{\infty}(\mathbb{C})$ and mean ergodic on $H_{v}^{0}(\mathbb{C})$ provided that $v(r) e^{r}$ is non-increasing in some interval $\left[r_{0}, \infty[\right.$. In particular, it is mean ergodic on $H_{a, 1}^{0}(\mathbb{C})$ for every $a \leq 0$.

(ii) If $r^{a} e^{-r}=O(v(r))$, with $a>1 / 2$, then $J$ is not power bounded on $H_{v}^{\infty}(\mathbb{C})$.

(iii) $J$ is uniformly mean ergodic on $H_{v}^{\infty}(\mathbb{C})$ if for some $\alpha>1, v(r) e^{\alpha r}$ is nonincreasing.

(iv) $J$ is not uniformly mean ergodic on $H_{v}^{0}(\mathbb{C})$ if for all $\beta>1, v(r) e^{\beta r}$ is increasing in some interval $\left[r_{0}, \infty[\right.$. In particular $J$ is not uniformly mean ergodic on $H_{a, 1}^{0}(\mathbb{C})$ for all $a \in \mathbb{R}$.

(v) If $r^{3 / 2} e^{-r}=O(v(r))$, then $J$ is not mean ergodic on $H_{v}^{0}(\mathbb{C})$. In particular, it is not mean ergodic in $H_{a, \alpha}^{0}(\mathbb{C})$ when $\alpha<1, a \in \mathbb{R}$.

Proof. (i) The first statement follows from the estimates of the norm of $J^{n}$ in Proposition 3.2

Moreover, for each $k \in \mathbb{N}$,

$$
\begin{gathered}
\left\|J^{n}\left(z^{k}\right)\right\|_{v}=\frac{k !}{(n+k) !}\left\|z^{n+k}\right\|_{v} \\
\leq v(0) e^{r_{0}} \frac{k !}{(n+k) !}\left\|z^{n+k}\right\|_{1}=v(0) e^{r_{0}} \frac{k !}{(n+k) !}\left(\frac{n+k}{e}\right)^{n+k} .
\end{gathered}
$$

By the Stirling formula, this implies that successive iterates tend to zero on the polynomials. As $J$ is power bounded and the polynomials are a dense subset, $\left(J^{n}(f)\right)_{n}$ converges to zero for each $f \in H_{v}^{0}(\mathbb{C})$. This implies that $\frac{1}{N} \sum_{n=1}^{N} J^{n}(f)$ also converges to 0 for each $f \in H_{v}^{0}(\mathbb{C})$ and $J$ is mean ergodic on $H_{v}^{0}(\mathbb{C})$.

(ii) As $\frac{\left\|z^{n}\right\|_{a, 1}}{n !}=O\left(\frac{\left\|z^{n}\right\|_{v}}{n !}\right)$ and the sequence $\left(\frac{\left\|z^{n}\right\|_{a, 1}}{n !}\right)_{n}$ for $a>1 / 2$ is unbounded, we conclude that $J$ is not power bounded by Lemma 2.4

(iii) The sequence $\left(\left\|J^{n}\right\|_{v}\right)_{n}$ tends to zero by Proposition 3.2 , therefore

$$
\left\|\frac{1}{N} \sum_{n=1}^{N} J^{n}\right\|_{v} \leq \frac{1}{N} \sum_{n=1}^{N}\left\|J^{n}\right\|_{v} \rightarrow 0 .
$$

Hence, $J$ is uniformly mean ergodic.

(iv) If for all $\beta>1, v(r) e^{\beta r}$ is increasing in some interval $\left[r_{0}, \infty\left[, \sigma_{v}(J) \supset \overline{\mathbb{D}}\right.\right.$. In particular $1 \in \sigma_{v}(J)$. The conclusion follows from Proposition 2.3 .

(v) By the Stirling formula, the sequence $\left(\frac{\left\|z^{n}\right\|_{3 / 2,1}}{n n !}\right)_{n}$ does not tend to zero and $\left\|z^{n}\right\|_{3 / 2,1}=O\left(\left\|z^{n}\right\|_{v}\right)$. By Lemma 2.4 (ii), $J$ is not mean ergodic on $H_{v}^{0}(\mathbb{C})$.

Corollary 3.6. The integration operator $J$ is uniformly mean ergodic on $H_{\alpha}^{\infty}(\mathbb{C})$ and $H_{\alpha}^{0}(\mathbb{C})$ if $\alpha>1$, and it is not mean ergodic on these spaces if $\alpha<1$. If $\alpha=1$, then $J$ is not mean ergodic on $H_{1}^{\infty}(\mathbb{C})$, and it is mean ergodic but not uniformly mean ergodic on $H_{1}^{0}(\mathbb{C})$. 
Proof. All the statements but one follow from Theorem 3.5. It remains to show that $J$ is not mean ergodic on $H_{1}^{\infty}(\mathbb{C})$. The space $H_{1}^{\infty}(\mathbb{C})$ is a Grothendieck-Banach space with the Dunford-Pettis property since it is isomorphic to $\ell_{\infty}$ by Galbis [1] or Lusky [20]. As $\left\|J^{n}\right\|_{1} / n \rightarrow 0$, we can apply [17, Theorem 8] or [18, Theorem 5] to conclude that $J$ is not mean ergodic in $H_{1}^{\infty}(\mathbb{C})$ because it is not uniformly mean ergodic by Theorem 3.5 (iv) and Proposition 2.3 .

3.2. The differentiation operator. For the differentiation operator we mainly restrict our attention to the spaces $H_{a, \alpha}^{\infty}(\mathbb{C})$ and $H_{a, \alpha}^{0}(\mathbb{C})$.

Proposition 3.7. The following holds for $a>0$ :

$$
\left\|D^{n}\right\|_{a, \alpha}=O\left(n !\left(\frac{e \alpha}{n-a}\right)^{n-a}\right)
$$

and

$$
n !\left(\frac{e \alpha}{n+a}\right)^{n+a}=O\left(\left\|D^{n}\right\|_{a, \alpha}\right) .
$$

For $a \leq 0$ :

$$
\left\|D^{n}\right\|_{a, \alpha} \approx n !\left(\frac{e \alpha}{n+a}\right)^{n+a}
$$

and the equality holds for $a=0$.

Proof. In case $a \leq 0$ the upper estimate follows from [2, Proposition 1]. For $a>$ 0 , we use Cauchy's formula for the $n$th derivative and the fact that $(1+r)^{a}$ is submultiplicative to get, for all $n \in \mathbb{N}$,

$$
\left|D^{n} f(z)\right|(1+|z|)^{a} \leq C n ! \frac{e^{\alpha(R+|z|)}(1+R)^{a}}{R^{n}}\|f\|_{a, \alpha}
$$

for some $C>0$. Taking the infimum over $R>0$, we get the estimate.

For the lower estimate we use that

$$
\left\|D^{n}\right\|_{a, \alpha} \geq \frac{n ! v(0)}{\left\|z^{n}\right\|_{a, \alpha}} \approx n !\left(\frac{e \alpha}{n+a}\right)^{n+a} .
$$

Proposition 3.8. The spectrum of $D$ satisfies

$$
\sigma_{a, \alpha}(D)=\alpha \overline{\mathbb{D}} \text {. }
$$

Proof. If $|\lambda|<\alpha$, the function $e_{\lambda}(z):=e^{\lambda z}$ belongs to $H_{a, \alpha}^{0}(\mathbb{C})$ and satisfies $D e_{\lambda}=$ $\lambda e_{\lambda}$. Therefore $D-\lambda I$ is not invertible, and thus $\alpha \mathbb{D} \subset \sigma_{a, \alpha}(D)$. On the other hand, the spectral radius $r(D)$ of $D$ satisfies $r(D)=\lim _{n \in \mathbb{N}}\left\|D^{n}\right\|^{1 / n}$. By the Stirling formula and the upper estimates for the norms in Proposition 3.7 $r(D) \leq \alpha$.

By [2, Proposition 4], $D-\lambda I$ is not surjective on $H_{a, \alpha}^{\infty}(\mathbb{C})$ or on $H_{a, \alpha}^{0}(\mathbb{C})$ for $|\lambda|=\alpha$. On the other hand, we get the following:

Proposition 3.9. Let $v$ be a weight such that $D$ is continuous on $H_{v}^{\infty}(\mathbb{C})$ and $v(r) e^{\alpha r}$ is non-increasing. If $|\lambda|<\alpha$, then the operator $D-\lambda I$ is surjective on $H_{v}^{\infty}(\mathbb{C})$, and it even has a continuous linear right inverse. The same holds on $H_{v}^{0}(\mathbb{C})$. In particular, if $|\lambda|<\alpha, D-\lambda I$ has a continuous right inverse on $H_{a, \alpha}^{\infty}(\mathbb{C})$. 
Proof. Denote by $e_{\lambda}(z):=e^{\lambda z}$ and by $M_{\lambda} f(z):=\left(e_{\lambda} f\right)(z)$. We have

$$
D M_{-\lambda}=e_{-\lambda}(D-\lambda I)
$$

and

$$
M_{\lambda} D=(D-\lambda I) M_{\lambda}
$$

Set $K_{\lambda}=M_{\lambda} J M_{-\lambda}$. More precisely,

$$
K_{\lambda} f(z)=e^{\lambda z} \int_{0}^{z} e^{-\lambda \xi} f(\xi) d \xi, f \in \mathcal{H}(\mathbb{C}) .
$$

We have

$$
(D-\lambda I) K_{\lambda}=(D-\lambda I) M_{\lambda} J M_{-\lambda}=M_{\lambda} D J M_{-\lambda}=M_{\lambda} M_{-\lambda}=I .
$$

It remains to show that for $|\lambda|<\alpha, K_{\lambda}: H_{v}^{\infty}(\mathbb{C}) \rightarrow H_{v}^{\infty}(\mathbb{C})$ and $K_{\lambda}: H_{v}^{0}(\mathbb{C}) \rightarrow$ $H_{v}^{0}(\mathbb{C})$ are continuous. Integrating along the segment between 0 and $z$, we get

$$
\begin{gathered}
v(|z|)\left|K_{\lambda} f(z)\right| \leq|z| v(|z|) \int_{0}^{1} e^{|\lambda||z|(1-t)}|f(t z)| d t \\
\leq|z| \int_{0}^{1} e^{|\lambda||z|(1-t)} e^{-\alpha(1-t)|z|} v(t z)|f(t z)| d t \\
\leq\|f\|_{v}|z| \int_{0}^{1} e^{(|\lambda|-\alpha)|z|(1-t)} d t \leq\|f\|_{v} \frac{1}{\alpha-|\lambda|}
\end{gathered}
$$

which finishes the proof for $H_{v}^{\infty}(\mathbb{C})$.

Now, since $K_{\lambda}: H_{v}^{0}(\mathbb{C}) \rightarrow H_{v}^{\infty}(\mathbb{C})$ is continuous and the polynomials are dense in $H_{v}^{0}(\mathbb{C})$, it is enough to show that $K_{\lambda}(\mathcal{P}) \subset H_{v}^{0}(\mathbb{C})$. To this end,

$$
K_{\lambda}(1)=-\frac{1}{\lambda}+\frac{1}{\lambda} e^{\lambda z} \in H_{v}^{0}(\mathbb{C})
$$

for $|\lambda|<\alpha$. Integrating by parts

$$
K_{\lambda}\left(z^{n}\right)=-\frac{1}{\lambda} z^{n}+\frac{n}{\lambda} K_{\lambda}\left(z^{n-1}\right), n \in \mathbb{N},
$$

from where the conclusion follows.

Proposition 3.10. (i) For $\alpha>1$ or $\alpha=1$ and $a<1 / 2$, $D$ is not power bounded on $H_{a, \alpha}^{\infty}(\mathbb{C})$.

(ii) If $v(r)=o\left(e^{-r}\right)$, then $D$ is not mean ergodic on $H_{v}^{0}(\mathbb{C})$. Consequently, $D$ is not mean ergodic on $H_{a, \alpha}^{0}(\mathbb{C})$ if $\alpha>1$ or if $\alpha=1$ and $a<0$.

(iii) For $\alpha<1, D$ is power bounded and uniformly mean ergodic on $H_{a, \alpha}^{\infty}(\mathbb{C})$.

(iv) $D$ is not uniformly mean ergodic on $H_{a, 1}^{0}(\mathbb{C}), a \in \mathbb{R}$.

Proof. (i) It is enough to observe that $\left\|D^{n}\right\|_{a, \alpha} \geq \frac{n ! v(0)}{\left\|z^{n}\right\|_{a, \alpha}}$, which tends to infinity by the Stirling formula.

(ii) If $D$ is mean ergodic on $H_{v}^{0}(\mathbb{C})$, for each $f \in H_{v}^{0}(\mathbb{C})$

$$
\frac{f^{\prime}+f^{\prime \prime}+\cdots+f^{(N)}}{N} \rightarrow 0,
$$

which is not the case for $e^{z} \in H_{v}^{0}(\mathbb{C})$.

(iii) By Proposition 3.7.

$$
\left\|D^{n}\right\|_{a, \alpha}=O\left(n !\left(\frac{e \alpha}{n-|a|}\right)^{n-|a|}\right) .
$$


We apply the Stirling formula to get

$$
\left\|D^{n}\right\|_{a, \alpha}=O\left(\frac{(n)^{n-|a|}}{(n-|a|)^{(n-|a|)}} e^{-|a|} \alpha^{n-|a|} n^{|a|+1 / 2}\right) .
$$

Therefore, for $\alpha<1, \lim _{n \rightarrow \infty}\left\|D^{n}\right\|_{a, \alpha}=0$; hence

$$
\lim _{n \rightarrow \infty}\left\|\frac{1}{n} \sum_{k=1}^{n} D^{k}\right\|_{a, \alpha} \leq \lim _{n \rightarrow \infty} \frac{1}{n} \sum_{k=1}^{n}\left\|D^{k}\right\|_{a, \alpha}=0 .
$$

(iv) In this case, $1 \in \sigma_{a, 1}(D)$, and the conclusion follows from Proposition 2.3 .

Corollary 3.11. $D$ is not mean ergodic on $H_{1}^{\infty}(\mathbb{C})$.

Proof. The space $H_{1}^{\infty}(\mathbb{C})$ is a Grothendieck Banach space with the Dunford-Pettis property. In fact it is isomorphic to $\ell_{\infty}$ by Galbis [11] or Lusky [20. Since the operator of differentiation satisfies $\left\|D^{n}\right\|_{1} / n \rightarrow 0$, we can apply [17, Theorem 8] or [18. Theorem 5] to conclude that $D$ is not mean ergodic on $H_{1}^{\infty}(\mathbb{C})$ because it is not uniformly mean ergodic by Propositions 3.10 (iv) and 2.3

We do not know if the differentiation operator is mean ergodic on the space $H_{1}^{0}(\mathbb{C})$. Related partial results can be seen in $[7$.

\subsection{The Hardy operator.}

Theorem 3.12. Let $v$ be an arbitrary weight. The Hardy operator $H: H_{v}^{\infty}(\mathbb{C}) \rightarrow$ $H_{v}^{\infty}(\mathbb{C})$ is well defined and continuous with norm $\|H\|_{v}=1$. Moreover, $H^{2}\left(H_{v}^{\infty}(\mathbb{C})\right)$ $\subset H_{v}^{0}(\mathbb{C})$ and $H^{2}$ is compact. If the integration operator $J: H_{v}^{\infty}(\mathbb{C}) \rightarrow H_{v}^{\infty}(\mathbb{C})$ is continuous, then $H$ is compact.

Proof. For every $f \in H_{v}^{\infty}(\mathbb{C})$ and $z \in \mathbb{C}$ we have

$$
v(|z|)\left|\frac{1}{z} \int_{0}^{z} f(\omega) d \omega\right| \leq v(|z|) \sup _{|\omega|=z}|f(\omega)| .
$$

Hence, $\|H\|_{v} \leq 1$. On the other hand, since $H(c)=c$ for every $c \in \mathbb{C}$, taking $g:=1 / v(0) \in H_{v}^{0}(\mathbb{C}),\|g\|_{v}=1$, we obtain $\|H\|_{v}=1$.

Given $f=\sum_{k=0}^{\infty} a_{k} z^{k} \in H_{v}^{\infty}(\mathbb{C})$, the Cauchy inequalities imply $\left|a_{k}\right|\left\|z^{k}\right\|_{v} \leq$ $\|f\|_{v}$ for every $k \in \mathbb{N}_{0}$. Then, as $H^{2}(f)(z)=\sum_{k=0}^{\infty} \frac{a_{k}}{(k+1)^{2}} z^{k}$, one has

$$
\left\|H^{2}(f)-\sum_{k=0}^{N} \frac{a_{k}}{(k+1)^{2}} z^{k}\right\|_{v} \leq \sum_{k=N+1}^{\infty} \frac{\left|a_{k}\right|\left\|z^{k}\right\|_{v}}{(k+1)^{2}} \leq\|f\|_{v} \sum_{k=N+1}^{\infty} \frac{1}{(k+1)^{2}} .
$$

Therefore, $H^{2}(f)$ belongs to the closure of the polynomials, and thus to $H_{v}^{0}(\mathbb{C})$.

The argument above also shows that the finite rank operators $H_{N}^{2}\left(\sum_{k=0}^{\infty} a_{k} z^{k}\right):=$ $\sum_{k=0}^{N} \frac{a_{k}}{(k+1)^{2}} z^{k}$ are bounded on $H_{v}^{\infty}(\mathbb{C})$ and that

$$
\left\|H^{2}-H_{N}^{2}\right\|_{v} \leq \sum_{k=N+1}^{\infty} \frac{1}{(k+1)^{2}}
$$

from where the compactness of $H^{2}$ follows. 
Finally, suppose that the integration operator $J: H_{v}^{\infty}(\mathbb{C}) \rightarrow H_{v}^{\infty}(\mathbb{C})$ is continuous. Then the Hardy operator $H: H_{v}^{\infty}(\mathbb{C}) \rightarrow H_{v}^{0}(\mathbb{C})$ is well defined, since for every $z \in \mathbb{C}, z \neq 0$,

$$
v(|z|)|H(f)(z)|=v(|z|) \frac{1}{|z|}|J(f)(z)| \leq \frac{\|J\|}{|z|}\|f\|_{v} .
$$

From here we also conclude that given a bounded set $B \subseteq H_{v}^{\infty}(\mathbb{C})$ and $\epsilon>0$, there exists $R>0$ such that for every $f \in B$ and $|z|>R, v(|z|)|H(f)(z)|<\epsilon$, and the conclusion follows by [22, Lemma 2.1].

By the work of Harutyunyan and Lusky [13, the integration operator $J$ is not continuous on $H_{v}^{\infty}(\mathbb{C})$ for $v(r)=\exp \left(-(\log r)^{2}\right)$. Moreover, by Lusky 19, Theorem 2.5.], the monomials constitute a basis of the space $H_{v}^{0}(\mathbb{C})$ and the norm of $H_{v}^{\infty}(\mathbb{C})$ is equivalent to $\left\|\sum_{k=0}^{\infty} a_{k} z^{k}\right\|_{v}=\sup _{k}\left|a_{k}\right|\left\|z^{k}\right\|_{v}$. Moreover, $H_{v}^{0}(\mathbb{C})$ is isomorphic to $c_{0}$. In this example the Hardy operator $H$ maps $H_{v}^{\infty}(\mathbb{C})$ into $H_{v}^{0}(\mathbb{C})$ (just look at the Taylor expansion of the function). As $H_{v}^{\infty}(\mathbb{C})$ is canonically isometric to the bidual of $H_{v}^{0}(\mathbb{C})$ by [4] or [6], and $H$ is weakly compact as an operator on both spaces $H_{v}^{\infty}(\mathbb{C})$ and $H_{v}^{0}(\mathbb{C})$. Since $H_{v}^{0}(\mathbb{C})$ is isomorphic to $c_{0}, H$ is compact on $H_{v}^{0}(\mathbb{C})$ (see e.g. [14, Corollary 17.2.6]). As $H$ on $H_{v}^{\infty}(\mathbb{C})$ coincides with the bi-transpose, it follows that it is also compact.

Corollary 3.13. The operator $H$ is power bounded and uniformly mean ergodic on $H_{v}^{\infty}(\mathbb{C})$. Moreover, its spectrum is $\sigma(H)=\left\{\frac{1}{n}\right\}_{\mathbb{N}} \cup\{0\}$.

Proof. As $H$ is quasi-compact, $\sigma(H)=\overline{\{\lambda: \lambda \text { is an eigenvalue of } H\}}$ and the eigenvalues of $H$ are $\left\{\frac{1}{n}: n \in \mathbb{N}\right\}$. Clearly $H$ is power bounded. The compactness of $H^{2}$ implies that $\operatorname{Im}(I-H)$ is closed. Now the conclusion follows from a criterion due to Lin [16] (see also [15, Theorem 2.2.1]).

Observe that contrary to what happens for the operators of integration $J$ and of differentiation $D$, the Hardy operator $H$ is mean ergodic and 1 belongs to the spectrum of $H$ on the space $H_{v}^{0}(\mathbb{C})$. In this case, the Cesàro means of the iterates of $H$ do not converge to zero on the polynomials. Being power bounded, $H$ cannot be hypercyclic on $H_{v}^{0}(\mathbb{C})$. In fact, since $\delta_{0}\left(H^{n} f\right)=f(0)$ for each $f \in \mathcal{H}(\mathbb{C}), H$ is not hypercyclic on $\mathcal{H}(\mathbb{C})$.

\section{REFERENCES}

[1] A.G. Arvanitidis, A.G. Siskakis, Cesàro operators on the Hardy spaces of the half-plane, arXiv:1006.1520v1.

[2] Aharon Atzmon and Bruno Brive, Surjectivity and invariant subspaces of differential operators on weighted Bergman spaces of entire functions, Bergman spaces and related topics in complex analysis, Contemp. Math., vol. 404, Amer. Math. Soc., Providence, RI, 2006, pp. 27-39, DOI 10.1090/conm/404/07632. MR2244002 (2007j:47063)

[3] Frédéric Bayart and Étienne Matheron, Dynamics of linear operators, Cambridge Tracts in Mathematics, vol. 179, Cambridge University Press, Cambridge, 2009. MR2533318 $(2010 \mathrm{~m}: 47001)$

[4] Klaus D. Bierstedt, José Bonet, and Antonio Galbis, Weighted spaces of holomorphic functions on balanced domains, Michigan Math. J. 40 (1993), no. 2, 271-297, DOI 10.1307/mmj/1029004753. MR1226832(94i:46034)

[5] Klaus D. Bierstedt, José Bonet, and Jari Taskinen, Associated weights and spaces of holomorphic functions, Studia Math. 127 (1998), no. 2, 137-168. MR.1488148 (99a:46037)

[6] K.D. Bierstedt and W.H. Summers, Biduals of weighted Banach spaces of analytic functions, J. Austral. Math. Soc. Ser. A 54 (1993), no. 1, 70-79. MR.1195659 (94a:46027) 
[7] Oscar Blasco and Antonio Galbis, On Taylor coefficients of entire functions integrable against exponential weights, Math. Nachr. 223 (2001), 5-21, DOI 10.1002/15222616(200103)223:1<5::AID-MANA5>3.3.CO;2-F. MR 1817845 (2002f:30034)

[8] José Bonet, Dynamics of the differentiation operator on weighted spaces of entire functions, Math. Z. 261 (2009), no. 3, 649-657. MR2471093 (2010b:47023)

[9] José Bonet and Antonio Bonilla, Chaos of the differentiation operator on weighted Banach spaces of entire functions, Complex Anal. Oper. Theory 7 (2013), no. 1, 33-42. MR3010787

[10] José Bonet and Werner J. Ricker, Mean ergodicity of multiplication operators in weighted spaces of holomorphic functions, Arch. Math. (Basel) 92 (2009), no. 5, 428-437, DOI 10.1007/s00013-009-3061-1. MR2506944(2010d:47009)

[11] Antonio Galbis, Weighted Banach spaces of entire functions, Arch. Math. (Basel) 62 (1994), no. 1, 58-64, DOI 10.1007/BF01200439. MR.1249586 (94j:46028)

[12] Karl-G. Grosse-Erdmann and Alfredo Peris Manguillot, Linear chaos, Universitext, Springer, London, 2011. MR2919812

[13] Anahit Harutyunyan and Wolfgang Lusky, On the boundedness of the differentiation operator between weighted spaces of holomorphic functions, Studia Math. 184 (2008), no. 3, 233-247, DOI 10.4064/sm184-3-3. MR.2369141 (2009j:46063)

[14] Hans Jarchow, Locally convex spaces, B. G. Teubner, Stuttgart, 1981. Mathematische Leitfäden. [Mathematical Textbooks]. MR632257 (83h:46008)

[15] Ulrich Krengel, Ergodic theorems, de Gruyter Studies in Mathematics, vol. 6, Walter de Gruyter \& Co., Berlin, 1985. With a supplement by Antoine Brunel. MR.797411 (87i:28001)

[16] Michael Lin, On the uniform ergodic theorem, Proc. Amer. Math. Soc. 43 (1974), 337-340. MR0417821(54 \#5869)

[17] Heinrich P. Lotz, Tauberian theorems for operators on $L^{\infty}$ and similar spaces, Functional analysis: surveys and recent results, III (Paderborn, 1983), North-Holland Math. Stud., vol. 90, North-Holland, Amsterdam, 1984, pp. 117-133, DOI 10.1016/S0304-0208(08)71470-1. MR:761376 (86h:47062)

[18] Heinrich P. Lotz, Uniform convergence of operators on $L^{\infty}$ and similar spaces, Math. Z. 190 (1985), no. 2, 207-220, DOI 10.1007/BF01160459. MR797538(87e:47032)

[19] Wolfgang Lusky, On the Fourier series of unbounded harmonic functions, J. London Math. Soc. (2) 61 (2000), no. 2, 568-580, DOI 10.1112/S0024610799008443. MR 1760680 (2001c:46047)

[20] Wolfgang Lusky, On the isomorphism classes of weighted spaces of harmonic and holomorphic functions, Studia Math. 175 (2006), no. 1, 19-45, DOI 10.4064/sm175-1-2. MR2261698 (2007f:30089)

[21] Reinhold Meise and Dietmar Vogt, Introduction to functional analysis, Oxford Graduate Texts in Mathematics, vol. 2, The Clarendon Press Oxford University Press, New York, 1997. Translated from the German by M. S. Ramanujan and revised by the authors. MR 1483073 (98g:46001)

[22] Alfonso Montes-Rodríguez, Weighted composition operators on weighted Banach spaces of analytic functions, J. London Math. Soc. (2) 61 (2000), no. 3, 872-884, DOI 10.1112/S0024610700008875. MR1766111 (2001j:47026)

[23] Walter Rudin, Functional analysis, McGraw-Hill Book Co., New York, 1973. McGraw-Hill Series in Higher Mathematics. MR0365062 (51 \#1315)

Instituto Universitario de Matemática Pura y Aplicada, Universitat Politècnica de València, E-46071 Valencia, Spain

E-mail address: mabelme@upv.es

Current address: Facultat de Magisteri, Universitat de València, E-46022 València, Spain

E-mail address: maria.jose.beltran@uv.es

Instituto Universitario de Matemática Pura y Aplicada, Universitat Politècnica de València, E-46071 Valencia, Spain

E-mail address: jbonet@mat.upv.es

Departamento de Análisis Matemático, Universidad de Valencia, E-46100 Burjassot, SPAIN

E-mail address: carmen.fdez-rosell@uv.es 